\title{
Accurate and Autonomous Star Acquisition Method for Star Sensor under Complex Conditions
}

\author{
Hao Zhang, Yanxiong Niu, Jiazhen Lu, and He Zhang \\ Department of Instrument Science and Opto-Electronics Engineering, Beihang University, Beijing, China \\ Correspondence should be addressed to Jiazhen Lu; ljzbuaa@163.com
}

Received 23 September 2016; Accepted 25 December 2016; Published 31 January 2017

Academic Editor: Ilhan Tuzcu

Copyright (C) 2017 Hao Zhang et al. This is an open access article distributed under the Creative Commons Attribution License, which permits unrestricted use, distribution, and reproduction in any medium, provided the original work is properly cited.

\begin{abstract}
Star sensor is a preferred attitude measurement device for its extremely high accuracy. Star acquisition is the essential and critical procedure, which is aiming at acquiring accurate star areas. However, degenerated acquisition results under complex conditions become one of the major restrictions for modern star sensor. In this paper, an accurate and autonomous star acquisition method is proposed. Mathematical morphology and variable thresholding are combined for accurate star extraction; motion PSF is estimated in frequency domain and nonlinear filter is adopted for star restoration. Accurate star acquisition can be achieved based on only one star image. Simulations and laboratory experiments are conducted for verification. Several existing methods are also reproduced for comparison. Acquisition results demonstrate that the proposed method is effective and an excellent performance can be achieved autonomously under complex conditions, along with more detected stars and improved acquisition accuracy.
\end{abstract}

\section{Introduction}

Star sensor is a promising attitude measurement device in celestial navigation for its high accuracy and excellent autonomy [1-3]. The main working procedures of star sensor include star acquisition, star centroiding, star identification, and attitude calculation. Star acquisition is an essential and crucial step, which is to obtain accurate star areas from star image. Both the accuracy and the amount of acquired stars have great influence on system performance. Under favorable working conditions, where the imaging background is clean and the vehicle is steady, star acquisition can be easily achieved and the location accuracy is high. However, in advanced applications such as high-resolution observation, deep space exploration, and defense tasks, star sensor must work under complex conditions [4-6]. Different types of interferences, such as Gaussian noise, single point noise, and uneven illumination are inevitable during imaging. These interferences will pollute the star image, making it difficult to detect stars form background and bringing in centroiding error. More remarkably, the excessively dispersed and trailed star energy caused by vibration and maneuvering will enlarge location error drastically. Consequently, the performance of star sensor will reduce or even become invalid due to the unsatisfied star acquisition results. Therefore, declined performance of star acquisition becomes one of the major constraints for further applications of star sensor.

To improve the star acquisition performance under undesirable conditions, several algorithms have been proposed. Arbabmir gave out an integrated scheme including noise reduction, star extraction, and star centroiding, which can improve the star acquisition accuracy under uneven illumination [7]. Zhou et al. introduced a star map denoising method via spare representation and dictionary learning [8]. Jiang et al. showed a robust and accurate star segmentation algorithm based on morphology, which can eliminate imaging noise effectively [9]. Considering deduced location accuracy of a motion blurred star, Sun et al. proposed a star acquisition method and the location accuracy is about 0.8 pixels under dynamic imaging condition of $0.8^{\circ} \%$ [10]. To further improve the dynamic performance, Sun et al. present a star restoration method with the assistance of MEMSgyros, and the centroiding accuracy is about 0.2 pixels at $2^{\circ}$ /s under clean background [11]. However, these methods can either eliminate only one type of interference or need the help of other devices. Under the influence of different 
types of interferences, they may fail in operation due to their inconformity with complex situations and the unreliability of assistant devices. Therefore, the algorithm for accurate and autonomous star acquisition under complex conditions is still eagerly needed and is of great significance for a star sensor.

In this paper, an accurate and autonomous star acquisition method under complex conditions is proposed. Mathematical morphology and variable thresholding are combined for accurate star extraction; the motion PSF is estimated in frequency domain and nonlinear filter is adopted for star restoration. The star energy can be extracted and recovered based on only one star image. Star images for testification are generated through simulation and laboratory experiment. Star acquisition is conducted using proposed method and several existing methods. Acquisition results show that the proposed method is effective and an excellent performance can be achieved autonomously along with more detected stars and improved acquisition accuracy.

\section{Star Measurement Model and Analysis}

2.1. Star Measurement Model. The basic star measurement model for a star sensor can be described using the pinhole model [1], as shown in Figure 1. The star spot is accumulated to a light spot during exposure time. Optical defocus is moderately implemented to get subpixel location accuracy of star spot. The $i$ th measurement star vector $v_{i}$ in imaging frame $\left(X_{S}, Y_{S}, Z_{S}\right)$ can be expressed using

$$
v_{i}=\frac{1}{\sqrt{x_{i}^{2}+y_{i}^{2}+f^{2}}}\left[\begin{array}{c}
-x_{i} \\
-y_{i} \\
f
\end{array}\right]
$$

where $f$ is the focal length of the optical system and $\left(x_{i}, y_{i}\right)$ is the star centroid.

2.2. Star Measurement Analysis. Under real imaging conditions, several interferences should be taken into consideration at the same time. Numerous studies have been conducted on star imaging noise, including detector noise and environment noise [12-15]. In this paper, single point noise, uneven background illumination, and Gaussian noise are taken into account: single point noise can represent the abnormal response of single pixel; uneven background illumination is a common imaging situation under the effect of stray light, moonlight, or sunlight, especially when the system is working on low orbit or endoatmosphere; other noises, such as dark current noise and quantization noise, can be summarized into Gaussian noise for their randomness. These noises together can describe most of the imaging noise for a star sensor. Motion is another important interference which will largely affect the star centroiding. Under the influence of maneuvering and vibration, star spot location will change and the star energy will be trailed. For the exposure time is relatively short, the speed of movement can be regarded as constant during exposure time.

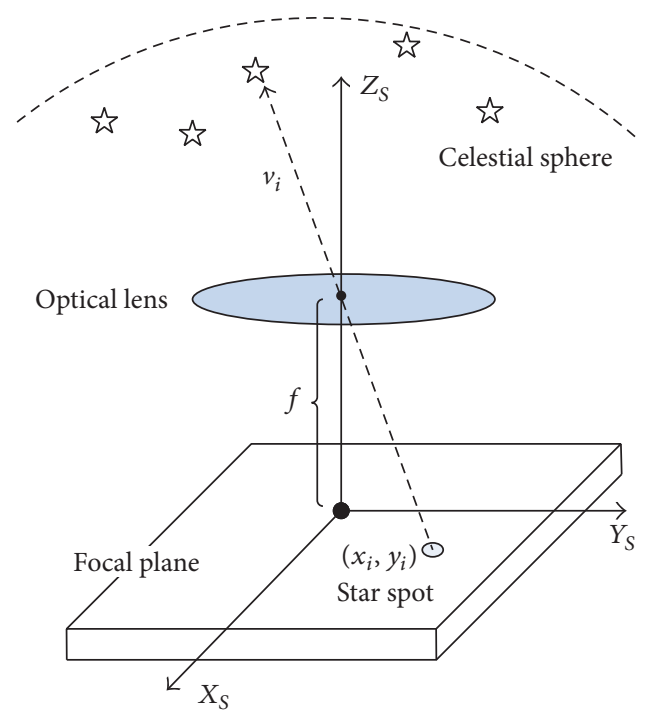

FIgURE 1: Star measurement model for star sensor.

Thus, the measured star vector is described as

$$
\begin{aligned}
v_{i}= & \frac{1}{\sqrt{\left(x_{i}+x_{i n}+x_{i m}\right)^{2}+\left(y_{i}+y_{i n}+y_{i m}\right)^{2}+f^{2}}} \\
& \cdot\left[\begin{array}{c}
-\left(x_{i}+x_{i n}+x_{i m}\right) \\
-\left(y_{i}+y_{i n}+y_{i m}\right) \\
f
\end{array}\right],
\end{aligned}
$$

where $\left(y_{i n}, x_{i n}\right)$ is the error caused by noise and $\left(y_{i m}, x_{i m}\right)$ is the error caused by movement, and

$$
\begin{aligned}
& x_{i m}=v_{x} t, \\
& y_{i m}=v_{y} t,
\end{aligned}
$$

where $v_{x}$ and $v_{y}$ are the velocity on $x$ and $y$ direction separately and $t$ is exposure time. Figure 2 shows the star measurement under complex conditions.

\section{Star Acquisition}

The core idea of accurate star acquisition is to distinguish the star energy from background and converge it into a reasonable area. The acquisition method is sketched as follows: the star extraction is carried out through combining morphological processing and variable thresholding; the motion PSF is estimated based on star image in frequency domain and nonlinear filter is utilized to star restoration. The working procedure is shown in Figure 3.

3.1. Star Extraction. Star extraction is aiming at extracting star area from background and is the prerequisite for star acquisition. Under influence of various interferences, we can hardly get satisfied results by an individual method. This paper combines morphological processing and variable thresholding to obtain accurate star area. 


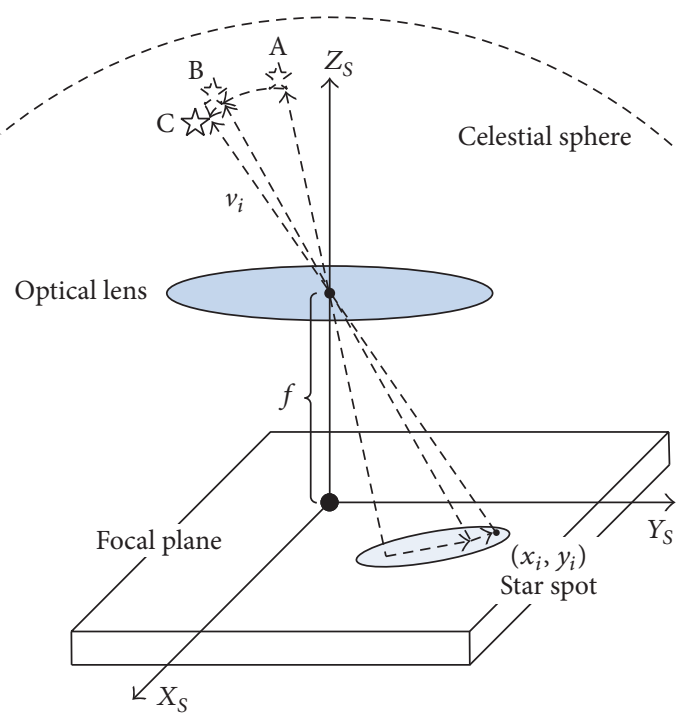

FIGURE 2: Star measurement model under complex conditions.

As analysed in Section 2, various noise may lead to decreased SNR and should be eliminated firstly. The most obvious specialty for noise pixels is that there exists saltation between noise and background. By meaning of finding out the saltation, the noise pixels can be distinguished. Isotropic filter, whose response is independent of the direction of the discontinuities in image, is widely used for border detection and can be suitable for preliminary noise pixel processing. The Laplacian Filter (LF) [16] is the simplest isotropic filter and is defined as

$$
\nabla^{2} f=\frac{\partial^{2} f}{\partial x^{2}}+\frac{\partial^{2} f}{\partial y^{2}}
$$

The original LF only takes the $x$ and $y$ directions into account; here we extend the original filter by adding second derivatives on $45^{\circ}$ and $135^{\circ}$ directions, respectively:

$$
\begin{aligned}
& \frac{\partial^{2} f}{\partial x y}=f(x-1, y+1)+f(x-1, y+1)-2 f(x, y) \\
& \frac{\partial^{2} f}{\partial y x}=f(x-1, y-1)+f(x+1, y+1)-2 f(x, y) .
\end{aligned}
$$

Then the template is isotropic on both axis and $45^{\circ}$ directions, with which we can get a better result than before. The Extended Laplacian Filter (ELF) can be expressed as

$$
\begin{aligned}
\nabla^{2} f(x, y)= & f(x-1, y-1)+f(x-1, y) \\
& +f(x-1, y+1)+f(x, y-1) \\
& +f(x, y+1)+f(x+1, y-1) \\
& +f(x+1, y)+f(x+1, y+1) \\
& -8 f(x, y),
\end{aligned}
$$

and the extended Laplacian operator is

$$
\nabla_{l}=\left[\begin{array}{ccc}
1 & 1 & 1 \\
1 & -8 & 1 \\
1 & 1 & 1
\end{array}\right] .
$$

The noise detection operation using ELF is

$$
f^{\prime}(x, y)=f(x, y)+c\left[\nabla_{l}^{2} f(x, y)\right],
$$

where $f(x, y)$ is the gray value of original image, $f^{\prime}(x, y)$ is the results, and $c$ is -1 .

After noise pixel detection, erosion is adopted for noise elimination. Erosion is a representative operation in morphology algorithm. The erosion can be expressed as

$$
\begin{aligned}
f_{e}(x, y) & =F^{\prime} \Theta b_{e}(x, y) \\
& =\min _{(m, n) \in D_{b_{e}}}\left[f^{\prime}(x+m, y+n)-b_{e}(m, n)\right],
\end{aligned}
$$

where $F^{\prime}$ represents the image after ELF operation, $b_{e}(m, n)$ is the structural element value of operator $b_{e}$ at pixel $(m, n)$, and $D_{b_{e}}$ is the domain of $b_{e}$. Since the sizes of noise pixels are smaller than $3 \times 3$ and the scale of stars spot is larger than 3 $\times 3$ pixels [17], the size of operator $b_{e}$ is set as 3 . With erosion operation, only star areas and gradually changed background are remaining in star image.

After noise elimination, a convenient way for star extraction is global thresholding. However, it may lead to large location error with the effect of uneven background. Under such situations, variable thresholding is an effective technic. Considering the request for real-time performance and stability, a variable thresholding using moving averages is adopted in this paper.

The moving average $m_{a}$ on pixel $(x, y)$ is

$$
\begin{aligned}
m_{a}(x, y)= & \frac{1}{l} \sum_{i=y+1-n}^{y} f(x, i) \\
= & m_{a}(x, y-1) \\
& +\frac{1}{l}(f(x, y)-f(x, y-1-l)),
\end{aligned}
$$

where $l$ is the length for calculating the average. The scanning of star image is carried out line by line. To avoid invalid operations near the edge, the borders of image are padded with $(l-1)$ zeros.

For star sensor, the size of a star spot is about $3 \times 3 \sim 9$ $\times 9$ pixels and the width will be within 9 pixels even for blurred stars. Meanwhile, the size of a detector is 1024 or larger and there will be no severe background changes within several pixels. According to analysis above and parameters of our facilities, the length $l$ is set as 50 . Additionally, the moving average may seem to be time-consuming. Fortunately, modern FPGA are good at logical and arithmetic operations, making it easy to achieve fast calculation of (10) on hardware.

At last, the thresholding matrix $T$ is expressed as

$$
T(x, y)=b * m_{a}(x, y),
$$

where $b$ is the adjustment factor and is set as 0.8 . 


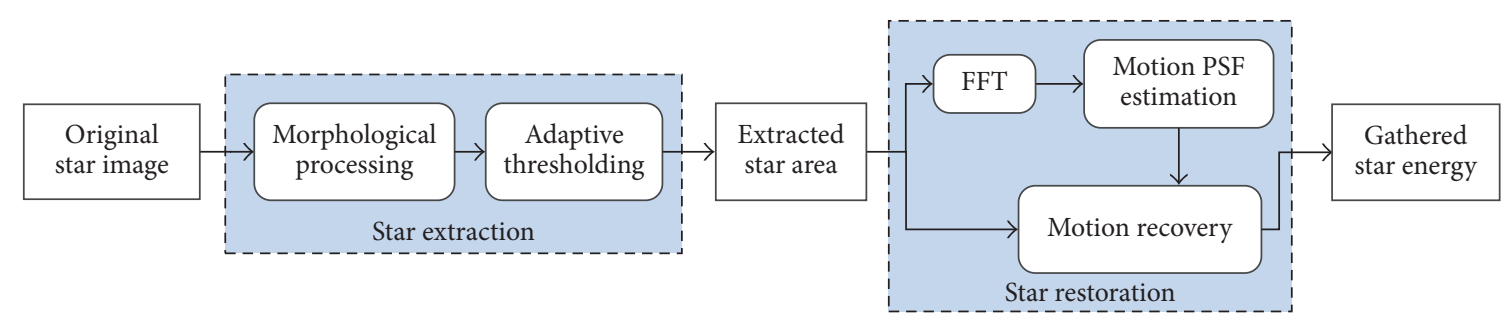

FIgURE 3: Star acquisition flow diagram.

3.2. Star Restoration. Under dynamic imaging conditions, the star area is dragged and the centroiding error will be unacceptable without any restoration or compensation. Several algorithms have been proposed for star restoration or motion compensation [11, 18, 19]. However, angular velocity information provided by gyros is indispensable in these algorithms, which complicates the navigation system and blemishes the autonomy of star sensor. To solve this problem, star restoration algorithm based on one star image is proposed in this paper. The restoration contains motion PSF estimation and restoration algorithm.

3.2.1. PSF Estimation Based on Radon Transformation in Frequency Domain. Radon transformation for 2D image can be defined as [20]

$$
\begin{aligned}
R & (\theta, \rho) \\
\quad & =\int_{y} \int_{x} f(x, y) \delta(\rho-x \cos \theta-y \cos \theta) d x d y,
\end{aligned}
$$

where $\rho$ is the distance between original point and line $L(\theta, \rho), \theta$ is the angle between $\rho$ and $x$ direction, $\rho=x \cos \theta+$ $y \cos \theta$, and $\delta$ is the impulse function.

The value of Radon transformation is the image projection on $L(\theta, \rho)$, as shown in Figure 4.

When $\theta$ is from $0^{\circ}$ to $180^{\circ}$, projections on different directions are received. Then $\theta_{\max }$ making $R$ the max value is corresponding to the motion direction $\theta_{m}$ and the relationship is

$$
\theta_{m}=\theta_{\max }+90^{\circ} \text {. }
$$

The motion length is obtained in frequency domain. Considering the Fourier transformation of blurred star,

$$
\begin{aligned}
F(u, v)= & \int_{0}^{T} G(u, v) e^{-j 2 \pi\left(u x_{0}(t) / M+v y_{0}(t) / N\right)} d t \\
& +W(u, v),
\end{aligned}
$$

where $F$ is the blurred star image, $G$ is the ideal image, $W(u, v)$ is noise, $T$ is the exposure time, $x_{0}(t), y_{0}(t)$ are the motion function, and $M$ and $N$ are the pixel value on $x$ and $y$ direction separately.

And the Fourier transformation of PSF is

$$
H(u, v)=\int_{0}^{T} e^{-j 2 \pi\left(u x_{0}(t) / M+v y_{0}(t) / N\right)} d t .
$$

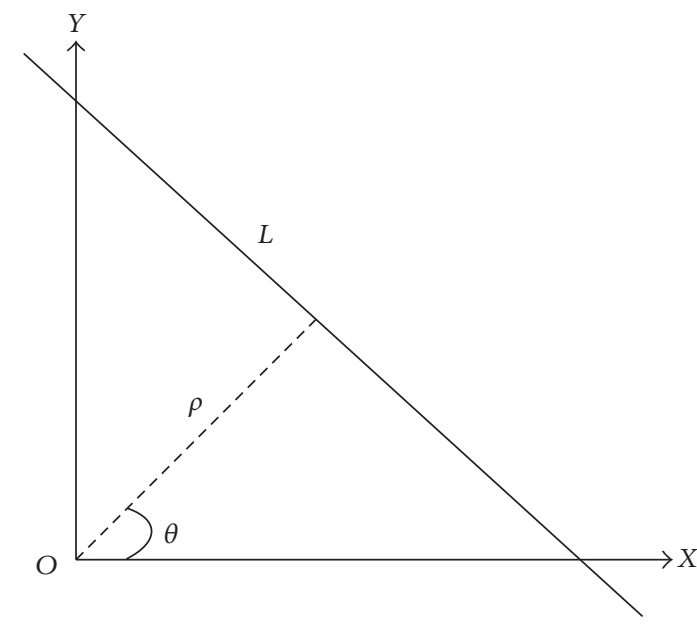

FIGURE 4: Radon transformation.

As the exposure time is short enough, the motion function can be regarded as uniform linear motion: $x_{0}(t)=a t / T$, $y_{0}(t)=b t / T$. Then the discretised results are

$$
\begin{aligned}
& |G(u, v)|=\left|\frac{T}{\pi(u a / M+v b / N)} \sin \left(\pi\left(\frac{u a}{M}+\frac{v b}{N}\right)\right)\right| \\
& \times|F(u, v)|+W(u, v), \\
& H(u, v)=\frac{T}{\pi(u a / M+v b / N)} \\
& \cdot \sin \left(\pi\left(\frac{u a}{M}+\frac{v b}{N}\right)\right) e^{-j \pi(u a / M+v b / N)} .
\end{aligned}
$$

It is obvious that $G$ and $\sin (\pi(u a / M+v b / N))$ have the same periodicity. So the value of $G$ will obtain fringes in frequency spectrum, and the width of fringe $d$ is

$$
d=\frac{1}{\sqrt{(a / M)^{2}+(b / N)^{2}}}
$$

where $a=L \cos \theta_{m}, b=L \sin \theta_{m}$, and $L$ is the motion length. For most star sensors, the detector has the same pixel value on $x$ and $y$ direction separately, so the motion length is

$$
L=\frac{M}{d \sqrt{\sin ^{2} \theta_{m}+\cos ^{2} \theta_{m}}} .
$$


In conclusion, the PSF can be calculated through following procedures: conduct Radon transformation and get its direction $\theta_{\max }$ where $R$ is maximum; the direction $\theta_{\max }+90^{\circ}$ is the motion direction; then conduct Fourier transformation and Radon transformation on $\theta_{\max }$ direction to get the width of fringe $d$; the motion length $L$ is calculated using formula (18).

\subsubsection{Star Restoration Filter Design Based on Lucy-Richardson} Algorithm. In modern restoration methods, linear restoration filters are widely used for their principle briefness and less calculation. However, nonlinear filters have the advantages of high restoration quality and noise restraining, and with the development of CPU, the shortage of complexity in calculation has reduced. So we use nonlinear filter here to complete the restoration filter design.

Lucy-Richardson (L-R) algorithm can convolve the image through interaction and calculate the maximum likelihood estimation as the restoration result with known PSF. The restoration filter is deduced as follow.

Considering Bayes formula

$$
\begin{aligned}
P(X \mid Y) & =\frac{P(Y \mid X) P(X)}{\int_{-\infty}^{+\infty} P(Y \mid X) P(X) d X}, \\
P(X) & =\int_{-\infty}^{+\infty} P(X \mid Y) P(Y) d Y,
\end{aligned}
$$

then $P(X)$ is

$$
P(X)=\int_{-\infty}^{+\infty} \frac{P(Y \mid X) P(Y)}{\int_{-\infty}^{+\infty} P(Y \mid X) P(X) d X} d Y P(X) .
$$

Used in star restoration, $P(X)$ represents restored star $f(x, y), P(Y)$ represents blurred star $g(\dot{x}, \dot{y}), P(Y \mid X)$ represents the PSF $g(\dot{x}-x, \dot{y}-y)$ of $(x, y)$, and then,

$$
\begin{aligned}
& f(x, y) \\
& \quad=\iint \frac{h(\dot{x}-x, \dot{y}-y) g(\dot{x}, \dot{y})}{\iint h(\dot{x}-x, \dot{y}-y) f(x, y) d x d y} d x^{\prime} d y^{\prime} \\
& \quad \cdot f(x, y) .
\end{aligned}
$$

Transfer to digital domain

$$
\begin{aligned}
& f(x, y) \\
& \quad=f(x, y)\left[h(-x,-y) * \frac{g(x, y)}{h(x, y) * f(x, y)}\right] .
\end{aligned}
$$

Rewrite (22) using iteration

$$
\begin{aligned}
& \widehat{f}_{k+1}(x, y) \\
& \quad=\widehat{f}_{k}(x, y)\left[h(-x,-y) * \frac{g(x, y)}{h(x, y) * \widehat{f}_{k}(x, y)}\right],
\end{aligned}
$$

where $\hat{f}_{k}(x, y)$ is the estimation on time $k$. Set the starting value as $\widehat{f}_{0}(x, y)=g(x, y)$; formula (23) is the restoration operation.

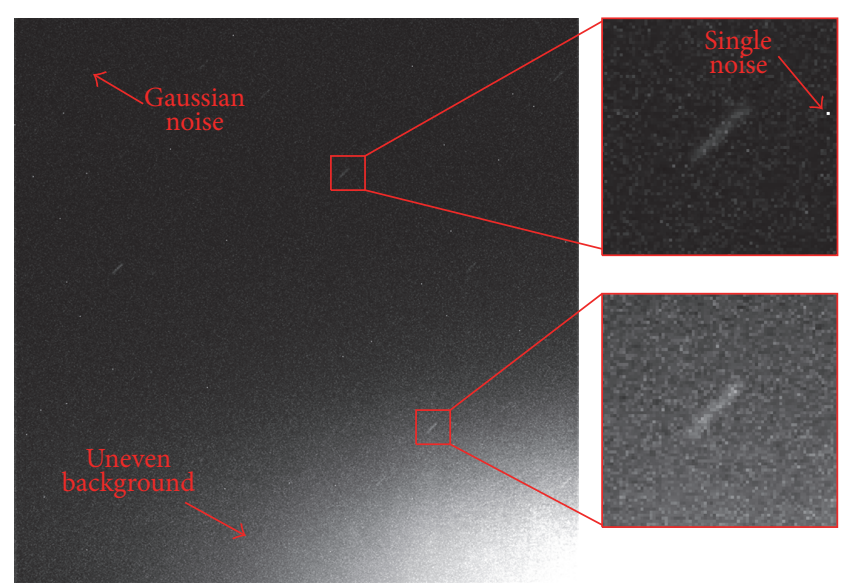

Figure 5: An example for simulated star images.

\section{Simulations and Experiments}

4.1. Simulation and Analysis. 20 star images are manufactured to test the proposed method. Original star images are acquired in Starry Night Pro, which is a powerful and professional astronomy software. The star camera parameters and observation conditions are shown in Table 1. To make the star image as realistic as possible, different types of interferences are added to captured images, as listed in Table 2. A typical image is shown in Figure 5. It can be seen that the star energy distribution is largely influenced by interferences. Part of star energy is submerged in noise and the SNR of stars is also different within one image under uneven background. What is more, the star energy is excessively tailed under dynamic conditions, adding large location error for accurate star acquisition.

Star acquisition is conducted using proposed method. Sun et al's method [10] is also reproduced for comparison. Considering the absence of consideration for dynamic condition in Arbabmir et al's method [7], we combine Arbabmir et al.s method and Sun et al.s method as another comparison: Arbabmir et al.s method is adopted for star extraction, followed by star restoration algorithm described in Sun et al.'s method. In the two comparisons, the precision of MEMSgyros is set as $100^{\circ} / \mathrm{h}$, which is a typical value for nowadays products. All of the calculations in this paper are achieved using MATLAB (R2013a) in a PC with Intel Core Duo $3.20 \mathrm{GHz}, 4 \mathrm{~GB}$ RAM. The typical results of three methods are shown in Figure 6.

It can be seen from Figure 6 that all of the three methods can eliminate some interferences, even with different effects. In Sun et al's method, there remains much noise, especially the uneven background. Due to the uncleanness of star image, the restoration algorithm cannot perform well. Some noise pixels are magnified after restoration and may be wrongly identified as a star. In the combined method, most of the noise can be eliminated and the restoration results seem to be acceptable. However, under dynamic conditions, the star energy will not follow Gaussian distribution and the SNR is much lower than that under static conditions. What is more, 
TABLE 1: Parameters of star sensor and observation environment in simulation.

\begin{tabular}{lc}
\hline FOV $\left(^{\circ}\right)$ & $10 \times 10$ \\
\hline Focal length $(\mathrm{mm})$ & 52 \\
Resolution (pixel) & $2048 \times 2048$ \\
Size of pixel $(\mu \mathrm{m})$ & 12 \\
Exposure time $(\mathrm{ms})$ & 80 \\
Maximum star magnitude $(\mathrm{Mv})$ & 6 \\
Capture time & $10: 07: 05$ 26th September 2015 \\
Location & $39^{\circ} 55^{\prime}$ Latitude, 116 23' Longitude (Beijing) \\
Atmosphere effect & Activated \\
\hline
\end{tabular}

TABLE 2: Interference parameters of star images.

\begin{tabular}{lccc}
\hline Bias of Gaussian noise & Single point noise (probability on each pixel) & Uneven background & Dynamic condition $\left({ }^{\circ} / \mathrm{s}\right)$ \\
\hline 5 & $0.01 \%$ & Activated & $\omega=2\left(\omega_{x}=\sqrt{2}, \omega_{y}=\sqrt{2}, \omega_{z}=0\right)$ \\
\hline
\end{tabular}

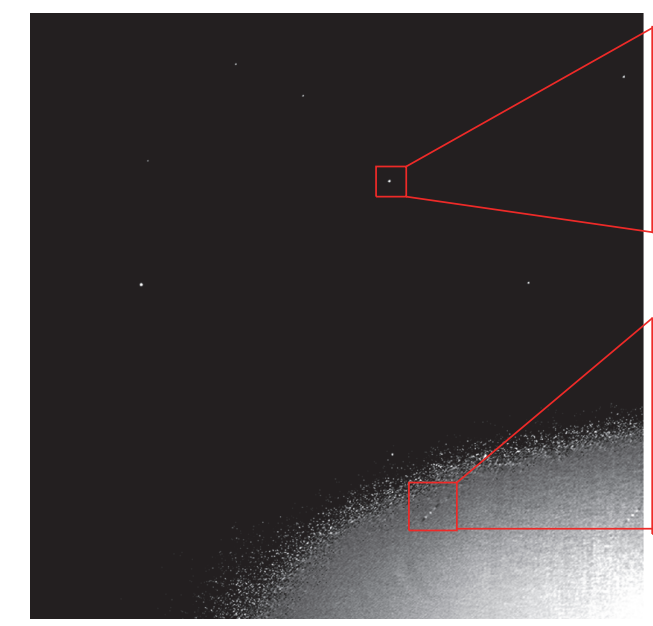

(a)

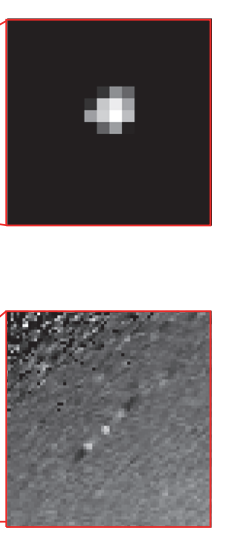

Undetected star

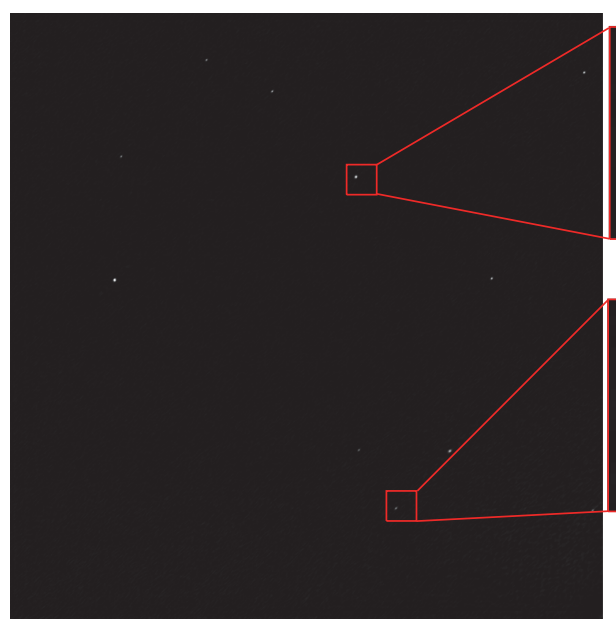

(b)

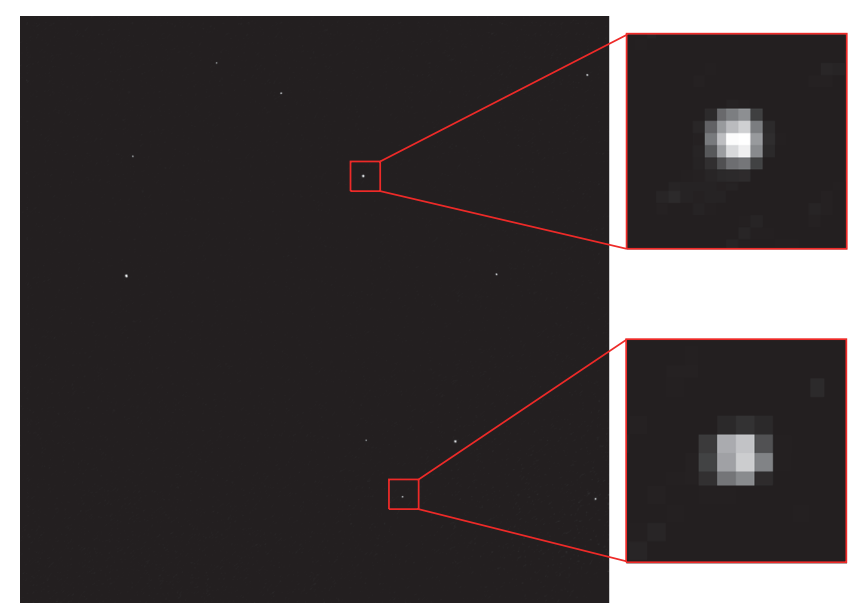

(c)

FIGURE 6: Typical star acquisition results. (a) Sun et al.s method; (b) the combined method; (c) proposed method. 


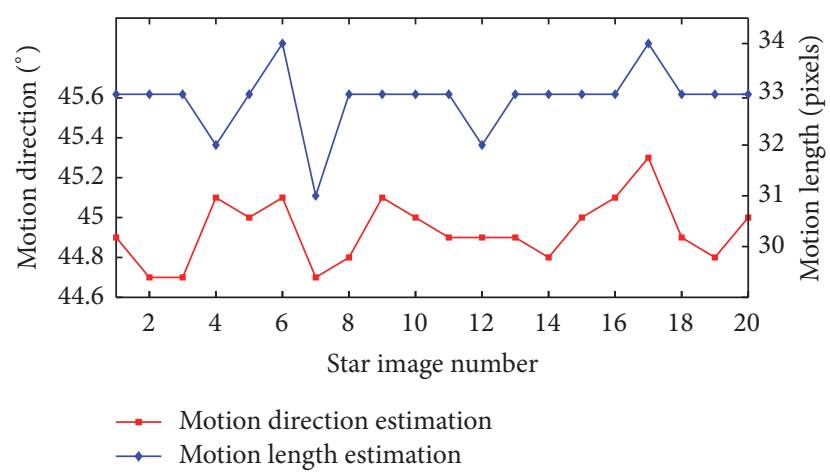

FIgURE 7: Motion PSF estimation results.

since Arbabmir et al's extraction algorithm is designed for static conditions, the blurred star energy distribution may be changed during extraction under dynamic conditions, resulting in insufficient restored energy distribution. It should be also known that both of the two methods cannot achieve star restoration with absence of angular rate information from gyros. In our method, external information is not needed during star acquisition. Different types of interferences are eliminated, bringing a clean background for star image. The star areas can be exactly extracted from background. Star energy can be restored and gathered within $3 \sim 9$ pixels, which is almost the same with star energy distribution under static conditions.

4.1.1. Total Number of Extracted Stars. To verify the superiority of our method on the quantity of star extraction, the total number of acquired stars is counted and shown in Table 3. The acquired ratios of Sun et al's method and the combined method are $77.49 \%$ and $94.53 \%$, respectively. They cannot satisfy the requirement for star identification. What is more, there exist 8 falsely detected stars in Sun et al's method, which may lead to failed star matching. The extracted ratio of proposed method is $99.04 \%$ with no falsely detected stars, which performs best in the three methods.

4.1.2. Motion PSF Estimation Accuracy. The accuracy of motion PSF estimation has a great influence on star restoration and should be discussed here. The estimation results of each star image are shown in Figure 7. The average value for estimated motion direction is $44.93^{\circ}$, and the error is $-0.15^{\circ}$ (in $1 \sigma$ ). The motion length on detector should be 32.8 pixels according to simulation settings. The average value for estimated motion length is 32.9 pixels, and the error is 0.64 pixels (in $1 \sigma$ ), which is less than 1 pixel. Since the resolution of restoration algorithm is 1 pixel on detector, our PSF estimation method can guarantee the restoration with high accuracy.

4.1.3. Star Acquisition Accuracy. To show the star acquisition accuracy, centroids of obtained stars are calculated using traditional weighted centroiding method. The true value of star location on imaging detector can be decided through coordinate transformation according to star catalogue and star

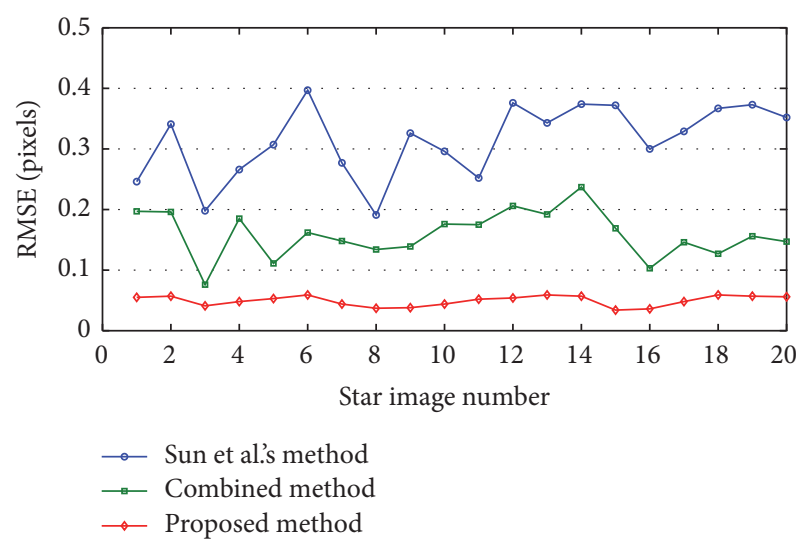

FIGURE 8: Star location error.

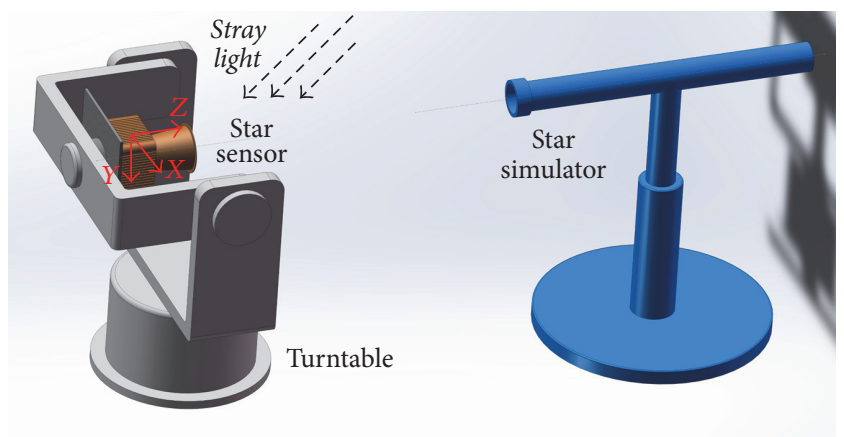

FIGURE 9: Experiment setup.

camera parameters. The Root Mean Square Error (RMSE) of extracted stars in each star image is shown in Figure 8. In the simulation, the centroiding error of proposed method is better than 0.06 pixels, which is much smaller than that of the other two methods.

In conclusion, compared with other methods, the proposed star acquisition method is proved to perform better in terms of interferences elimination, star extraction, star location, and autonomy.

4.2. Experiment and Analysis. Laboratory experiments are conducted for further verification. The experiment facilities include a 3-axis turntable, a single star simulator, and a star sensor. The parameters of star sensor are the same as those in the simulation and parameters of other facilities are listed in Table 4 . The star location error caused by these facilities (after calibration) is less than 0.01 pixels. The star sensor is fixed on the inner frame of the turntable and the initial optical axes of the star sensor and the star simulator are parallel. The experiment setup is shown in Figure 9. To arouse the noise of components of star sensor, the temperature of star sensor during experiment is about $38 \sim 50^{\circ} \mathrm{C}$, which is higher than ideal conditions. Stray light is added from one side of the star sensor.

4.2.1. Star Acquisition under Static Conditions. Star acquisition under static conditions is conducted firstly. Star images 
TABLE 3: Total number of extracted stars of each group.

\begin{tabular}{lcccc}
\hline & Total & Sun et al's method & Combined method & Proposed method \\
\hline Extracted & 311 & 241 & 294 & 308 \\
Falsely detected & $\backslash$ & 8 & 0 & 0 \\
\hline
\end{tabular}

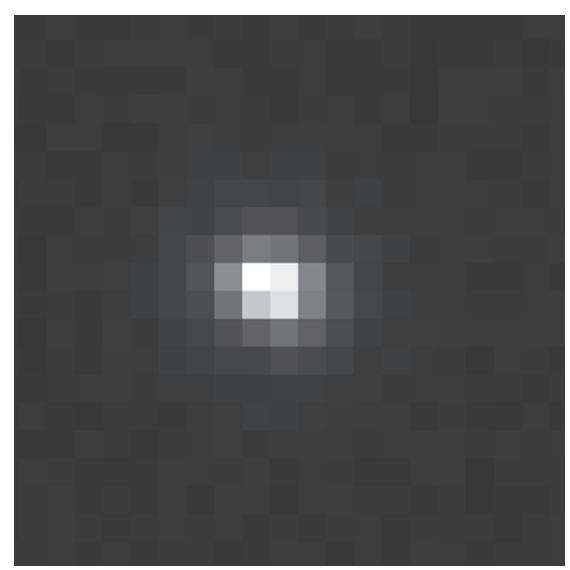

(a)

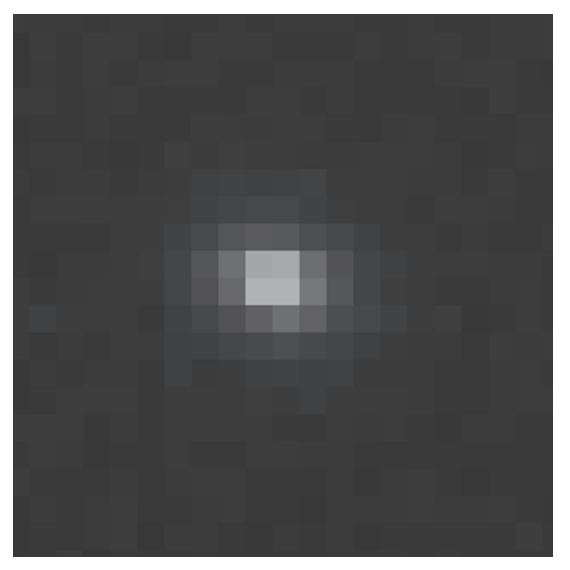

(d)

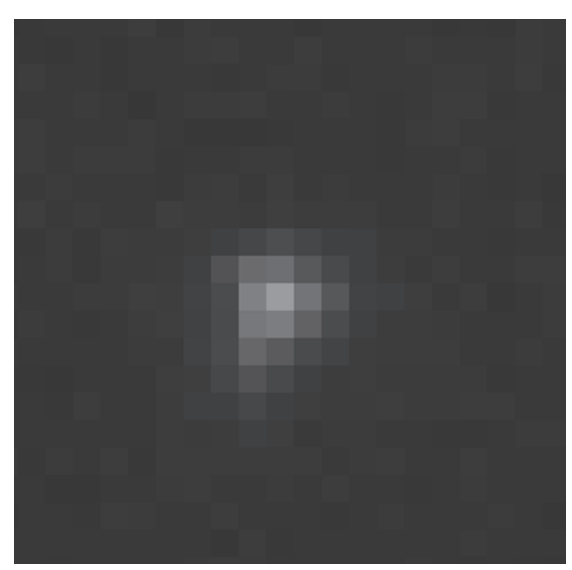

(g)

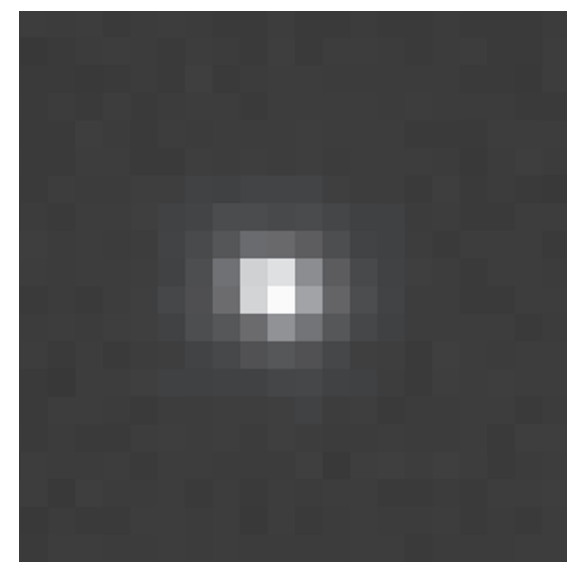

(b)

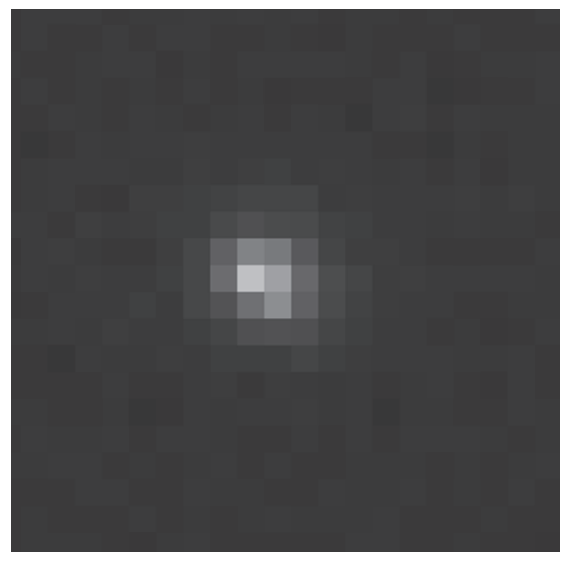

(e)

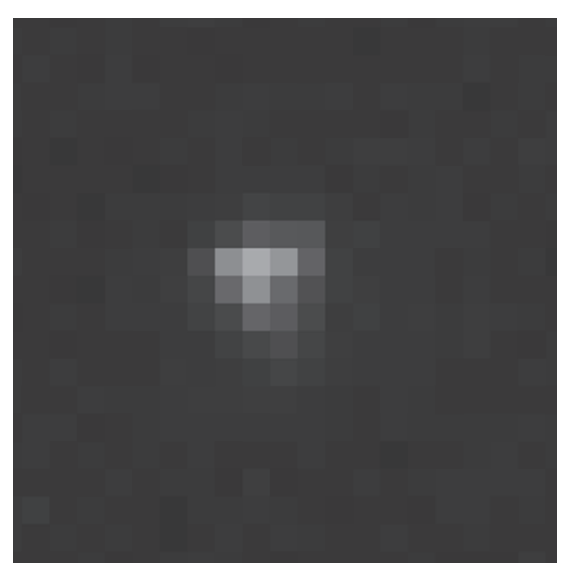

(h)

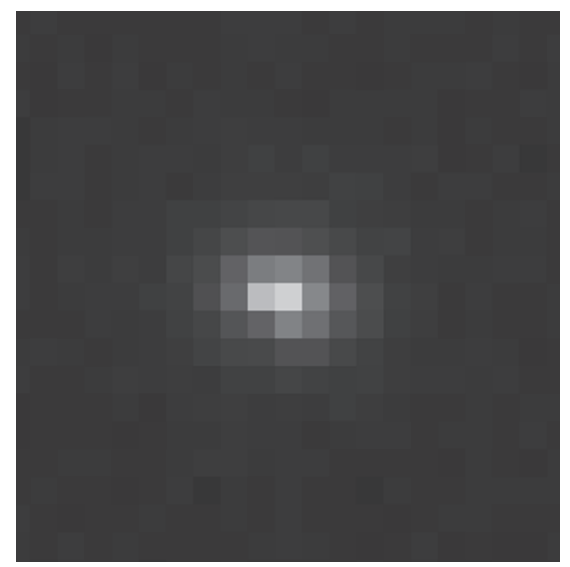

(c)

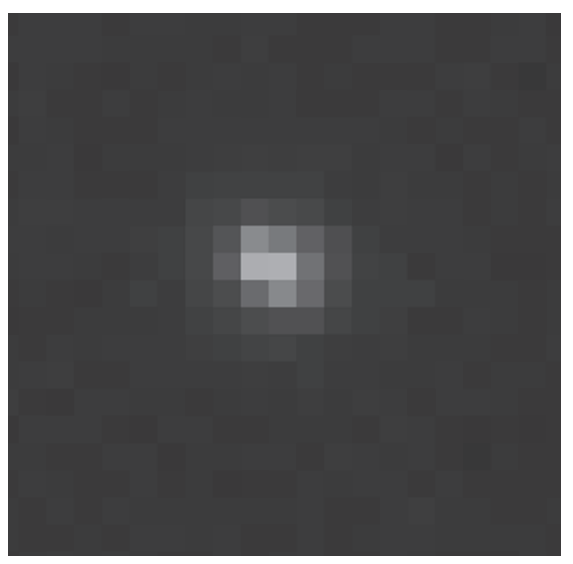

(f)

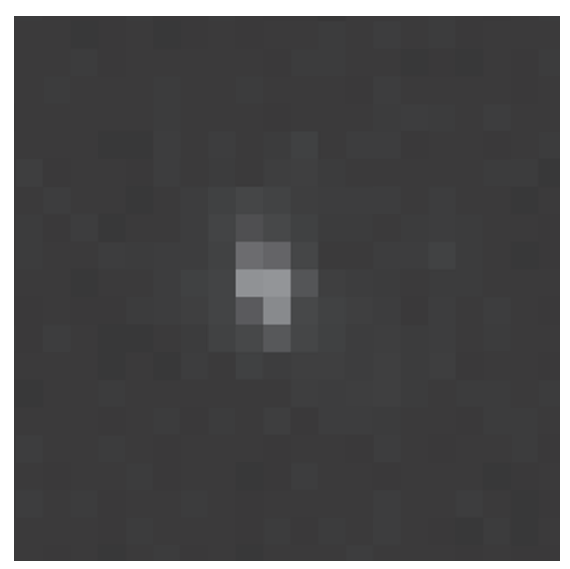

(i)

Figure 10: Typical stars under static conditions. The angles between the axis of star sensor and the axis of star simulator are (a) $-4^{\circ}$, (b) $-3^{\circ}$, (c) $-2^{\circ}$, (d) $-1^{\circ}$, (e) $0^{\circ}$, (f) $1^{\circ}$, (g) $2^{\circ},(\mathrm{h})-3^{\circ}$, and (i) $4^{\circ}$, respectively. 
TABle 4: Parameters of experiment facilities.

\begin{tabular}{lc}
\hline $\begin{array}{l}\text { Installation error between star sensor and turntable } \\
\text { (after calibration) }\end{array}$ & $\leq 1^{\prime \prime}$ \\
Turntable & \\
$\quad$ Pointing accuracy & $\leq 0.5^{\prime \prime}$ \\
$\quad$ Angular velocity error & $0.005 \%$ \\
Star simulator & \\
$\quad$ Star magnitude & $1 \mathrm{Mv}$ \\
$\quad$ Pointing accuracy & $\leq 0.5^{\prime \prime}$ \\
\hline
\end{tabular}

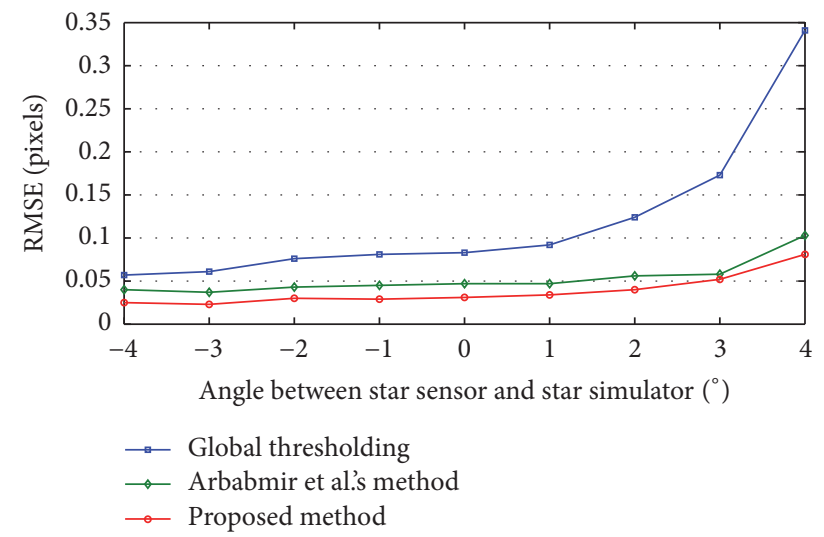

FIGURE 11: Star location error.

at different turntable positions are captured. A group of stars is shown in Figure 10. 10 star images are captured on each angular position. It can be seen from Figure 10 that, with the influence of stray light and uneven illumination, the brightness and energy distribution of observed star changes. In Figure 10(a), the star energy distribution is regular and the star is bright enough for star acquisition; in Figure 10(i), the energy distribution is abnormal and the star is dimmer than Figure 10(a).

The star acquisition is conducted using the global thresholding method, Arbabmir et al's method, and proposed method, followed by weighted centroiding method for star location calculation. The results are shown in Figure 11. For global thresholding method, the location accuracy can be easily influenced. The error is 0.13 pixels in average and can reach about 0.35 pixels under undesirable conditions in Figure 10(i). Arbabmir et al.s method has almost the same performance as ours under static conditions and the mean error is 0.05 pixels. The location error of proposed method is 0.04 pixels in average. The maximum of location error is 0.08 pixels, proving that our method can be hardly influenced by interferences.

4.2.2. Star Acquisition under Fixed Angular Rate. To further demonstrate the performance of proposed method, star images are obtained under dynamic conditions. Experiments under fixed angular rate are conducted. The angular rate is from $0.2^{\circ} \%$ to $2 \%$ and 10 star images are obtained under each condition. Star acquisition is achieved using proposed method. Several typical stars and the star acquisition results are shown in Figure 12. It is obvious that the original star is unavailable for star identification, for its centroiding error can be several pixels. The acquired stars using proposed method are much better than original ones. Most of the star energy is concentrated within about 7 pixels around the centroid, which is good for centroid calculation.

The PSF estimation results are shown in Figure 13(a), along with the statistics of restored energy within 7 pixels. The mean error of PSF estimation is 0.48 pixels. Under dynamic conditions of $2^{\circ} / \mathrm{s}$, the estimation error is 0.8 pixels, which is corresponding to the simulations results. It can be concluded that the PSF estimation accuracy is high enough under various dynamic conditions, which is the guarantee for accurate star restoration. Most of the star energy can be gathered around the centre, which is corresponding to static conditions. Figure 13(b) shows the mean star location error under different angular rates. Even influenced by motion, the accuracy is significantly improved. Under low dynamic conditions of $1^{\circ}$ s or less, the centroiding error is better than 0.08 pixels and the error is less than 0.12 pixels under high dynamic conditions of $2 \%$, which is at the same level with simulations and static experiments.

4.2.3. Star Acquisition under Varying Angular Rate. Experiments under varying angular rate are also conducted. The angular rate fits sine curve on $x$ direction, whose peak value is $2^{\circ} / \mathrm{s}$ and period is $10 \mathrm{~s}$. A typical star under about $1.8^{\circ}$ / s is shown in Figure 14(a) and its acquisition result is shown in Figure 14(b). The original and acquired star energy distribution on $x$ direction are shown in Figures 14(c) and $14(\mathrm{~d})$ separately. It is obvious that the original star is unavailable for star identification, for its centroiding error can be several pixels. The acquired star using proposed method is much better than original one. Most of the star energy is concentrated within about 7 pixels around the centroid, which is good for centroid calculation. The restored energy distribution fits idea Gaussian distribution well, leading to better centroiding accuracy.

Figure 15 illustrates the accuracy of restored star under varying dynamic conditions. It can be seen that the centroiding errors vary little during experiment, proving that the error caused by slew can be effectively eliminated using proposed method. Under relatively low angular rate, the centroiding error can be about $0.05 \sim 0.08$ pixels, which is corresponding to that under static conditions and fixed angular rate. The error can be still maintained within 0.15 pixels under $2^{\circ} \%$ or $1.26^{\circ} / \mathrm{s}^{2}$, showing the adaptability of proposed method. The statistics of centroiding error during the experiment is 0.062 pixels (in $1 \sigma$ ). The acquisition results are at the same level with that in simulations and fixed angular rates experiments.

In this section, various simulations and experiments are conducted for verification, followed by analysis and discussion. Both simulations and experiments results demonstrate the effectiveness, robustness, and high accuracy of proposed method in star acquisition. 

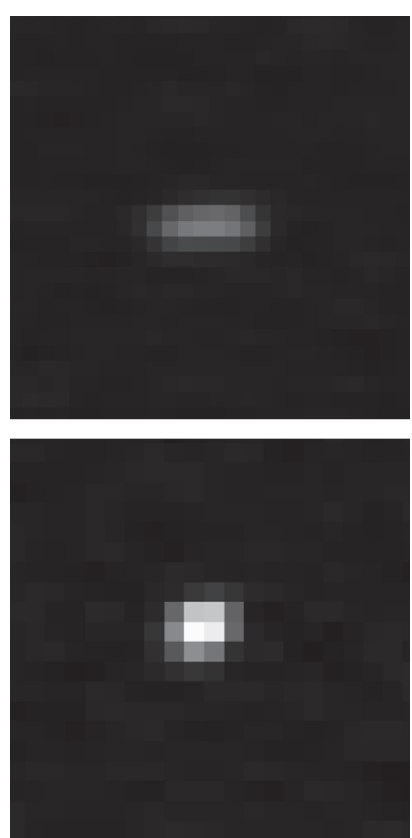

(a)
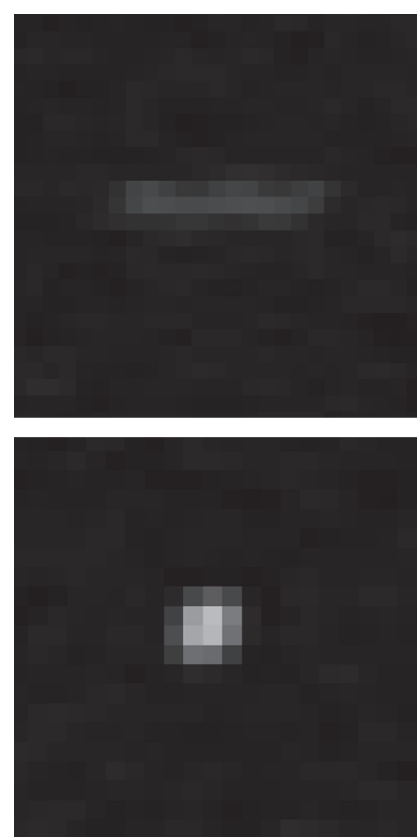

(b)
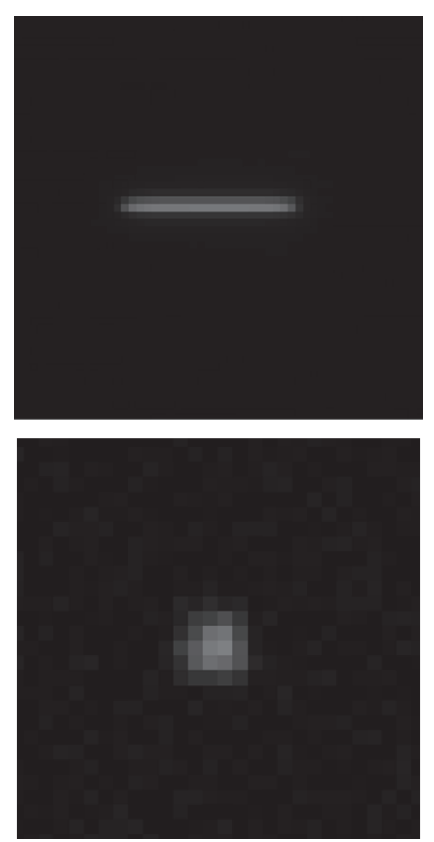

(c)
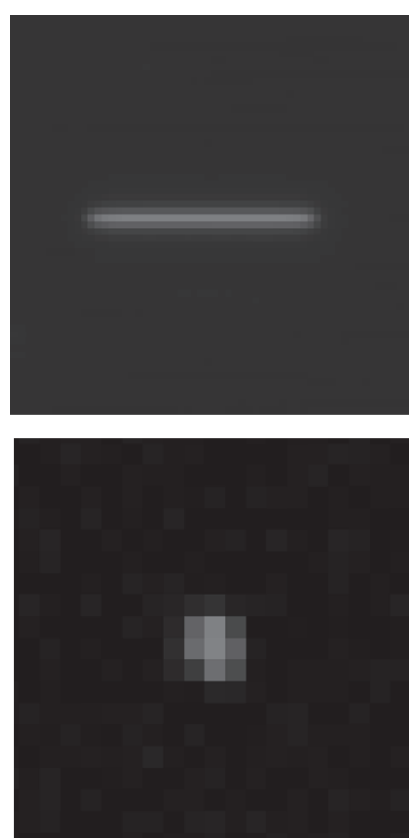

(d)

Figure 12: Typical stars and acquisition results under fixed angular rate. (a) A star under $0.4^{\circ} / \mathrm{s}$; (b) a star under $0.8^{\circ} / \mathrm{s}$; (c) a star under $1.4^{\circ} / \mathrm{s}$; (d) a star under $2.0^{\circ} / \mathrm{s}$.

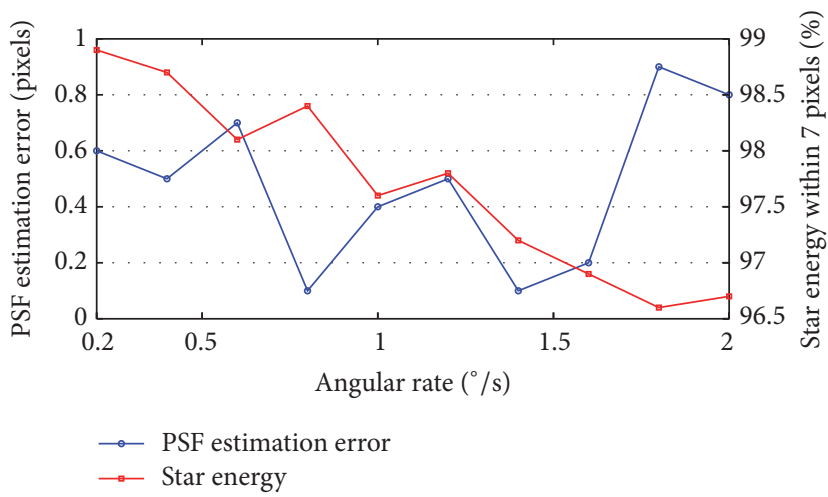

(a)

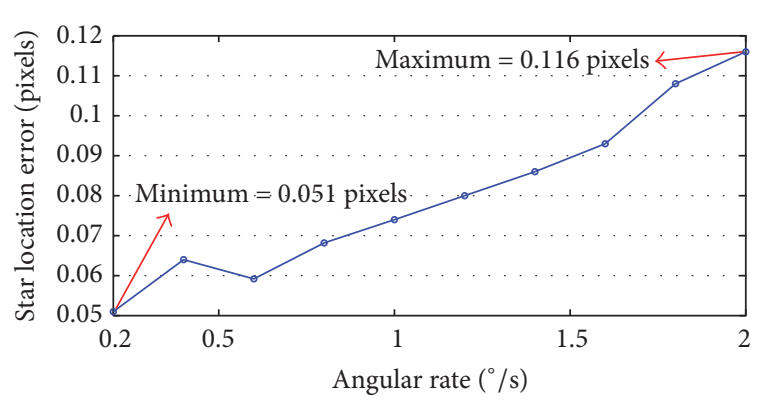

(b)

FIGURE 13: Star acquisition results under fixed angular rate. (a) PSF estimation results and star energy; (b) star location results.

\section{Conclusion}

A star acquisition method for star sensor under complex conditions is proposed in this paper. Mathematical morphology and variable thresholding are combined for accurate star extraction; motion PSF is estimated in frequency domain and nonlinear filter is adopted for star restoration. Accurate star acquisition can be achieved autonomously under complex imaging conditions. Star images are simulated to test our method. Star acquisition is conducted using proposed method and two other methods. Simulation results show that the star location error of proposed method is about 0.06 pixels, along with more extracted stars, which is much better than the other two methods. Laboratory experiment is conducted for further verification. Experiment results demonstrate that the location error can be better than 0.15 pixels under various dynamic conditions and the restored star energy fits the Gaussian distribution well. The proposed star acquisition method is proved to get an excellent performance in terms of acquired number, star location accuracy, and autonomy.

\section{Competing Interests}

The authors declare that they have no competing interests. 


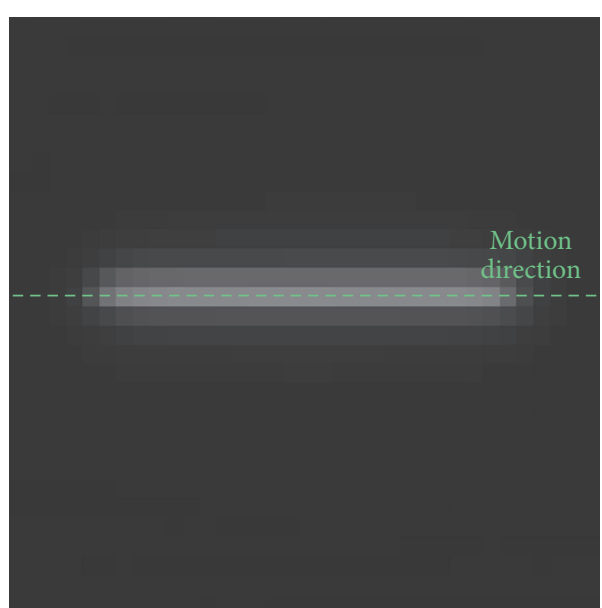

(a)

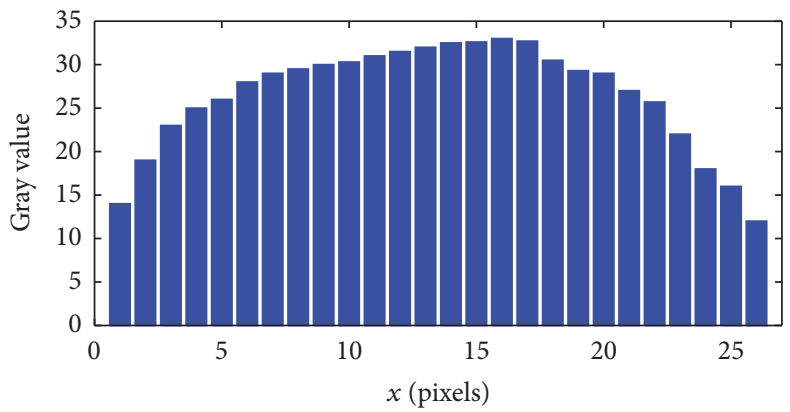

Gray value

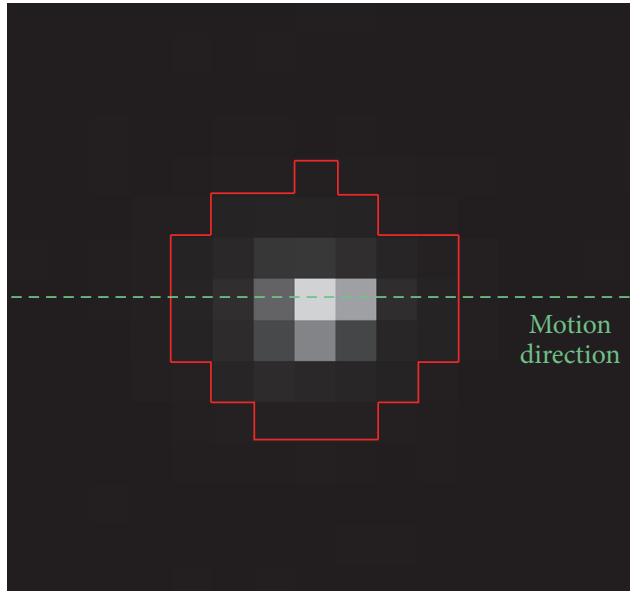

(b)

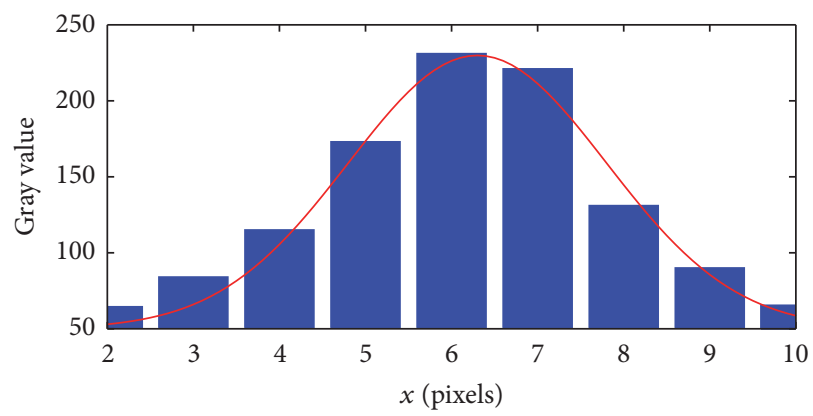

- Gray value

_ Ideal Gaussian distribution

(d)

Figure 14: A typical star under varying dynamic conditions. (a) Gray value of original star spot. (b) Gray value of acquired star spot using proposed method. (c) Energy distribution of original star. (d) Energy distribution of acquired star using proposed method.

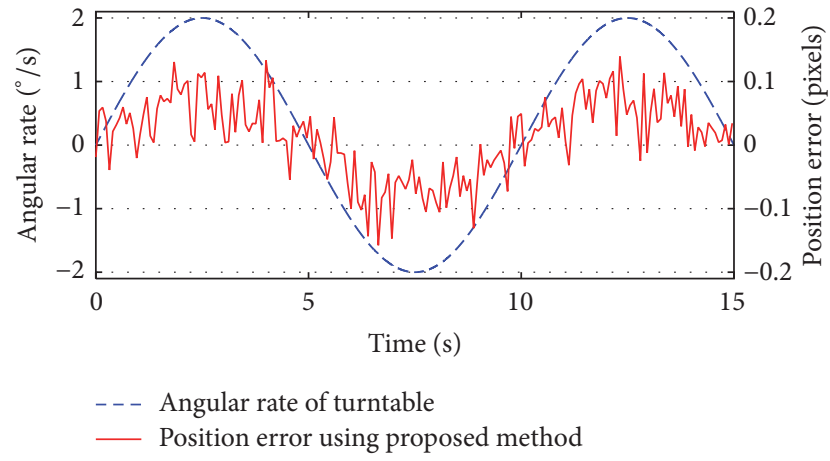

FIGURE 15: Star centroiding error in experiment.

\section{References}

[1] C. C. Liebe, "Accuracy performance of star trackers-a tutorial," IEEE Transactions on Aerospace and Electronic Systems, vol. 38, no. 2, pp. 587-599, 2002.

[2] C. C. Liebe, E. W. Dennison, B. Hancock, R. C. Stirbl, and B. Pain, "Active pixel sensor (APS) based star tracker," in Proceedings of the IEEE Aerospace Conference, vol. 111, pp. 119127, March 1998.
[3] U. Schmidt, C. Elstner, and K. Michel, "ASTRO 15 star tracker flight experience and further improvements towards the ASTRO APS star tracker," in Proceedings of the AIAA Guidance, Navigation and Control Conference and Exhibit, Honolulu, Hawaii, USA, August 2008.

[4] M. Samaan and S. Theil, "Development of a low cost star tracker for the SHEFEX mission," Aerospace Science and Technology, vol. 23, no. 1, pp. 469-478, 2012.

[5] R. W. H. van Bezooijen, K. A. Anderson, and D. K. Ward, "Performance of the AST-201 star tracker for the microwave anisotropy probe," in Proceedings of the AIAA Guidance, Navigation, and Control Conference and Exhibit, Monterey, Calif, USA, August 2002.

[6] D. Haley, T. Strikwerda, K. Ailinger, R. Casini, A. Landi, and R. Bettarini, "Star tracker scan mode capability for the new horizons mission," Acta Astronautica, vol. 542, pp. 299-306, 2003.

[7] M. V. Arbabmir, S. M. Mohammadi, S. Salahshour, and F. Somayehee, "Improving night sky star image processing algorithm for star sensors," Journal of the Optical Society of America A: Optics and Image Science, and Vision, vol. 31, no. 4, pp. 794801, 2014.

[8] M. Zhou, Y. Shi, and J. Yang, "Denoising star map data via sparse representation and dictionary learning," Optik, vol. 126, no. 1112, pp. 1133-1137, 2015. 
[9] J. Jiang, L. Lei, and Z. Guangjun, "Robust and accurate star segmentation algorithm based on morphology," Optical Engineering, vol. 55, no. 6, Article ID 063101, 2016.

[10] T. Sun, F. Xing, Z. You, and M. Wei, "Motion-blurred star acquisition method of the star tracker under high dynamic conditions," Optics Express, vol. 21, no. 17, pp. 20096-20110, 2013.

[11] T. Sun, F. Xing, Z. You, X. Wang, and B. Li, "Smearing model and restoration of star image under conditions of variable angular velocity and long exposure time," Optics Express, vol. 22, no. 5, pp. 6009-6024, 2014.

[12] X. Wei, W. Tan, J. Li, and G. Zhang, "Exposure time optimization for highly dynamic star trackers," Sensors, vol. 14, no. 3, pp. 4914-4931, 2014.

[13] J. Jiang, J. Li, J. Liu, Z. Hao, O. Y. Nosach, and J. Wang, "Active pixel sensor geometrical characteristic effects on star image subdivided locating accuracy for star tracker," in ICO20: Remote Sensing and Infrared Devices and Systems, 60310C, vol. 6031 of Proceedings of SPIE, February 2006.

[14] A. E. Gamal, H. Eltoukhy, and K. Salama, Cmos Sensors for Optical Molecular Imaging, Springer, New York, NY, USA, 2006.

[15] J. S. Lee, R. I. Hornsey, and D. Renshaw, "Analysis of CMOS photodiodes-Part I: quantum efficiency," IEEE Transactions on Electron Devices, vol. 50, no. 5, pp. 1233-1238, 2003.

[16] R. C. Gonzalez and R. E. Woods, Digital Image Processing, Addison-Wesley Longman, Boston, Mass, USA, 2001.

[17] H. Jia, J. Yang, X. Li et al., "Systematic error analysis and compensation for high accuracy star centroid estimation of star tracker," Science China Technological Sciences, vol. 53, no. 11, pp. 3145-3152, 2010.

[18] C.-S. Liu, L.-H. Hu, G.-B. Liu, B. Yang, and A.-J. Li, "Kinematic model for the space-variant image motion of star sensors under dynamical conditions," Optical Engineering, vol. 54, no. 6, Article ID 063104, 2015.

[19] Z. Jun, H. Yuncai, W. Li, and L. Da, "Studies on dynamic motion compensation and positioning accuracy on star tracker," Applied Optics, vol. 54, no. 28, pp. 8417-8424, 2015.

[20] M. E. Moghaddam, M. Jamzad, and H. R. Mahini, "Motion blur identification in noisy images using feed-forward back propagation neural network," in Advances in Machine Vision, Image Processing, and Pattern Analysis: International Workshop on Intelligent Computing in Pattern Analysis/Synthesis, IWICPAS 2006 Xian, China, August 26-27, 2006 Proceedings, vol. 4153 of Lecture Notes in Computer Science, pp. 369-376, Springer, Berlin, Germany, 2006. 


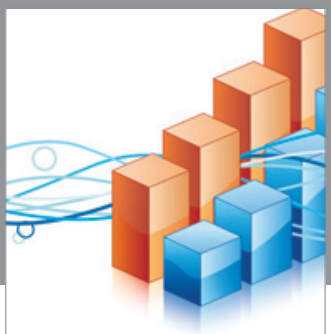

Advances in

Operations Research

vatem alat4

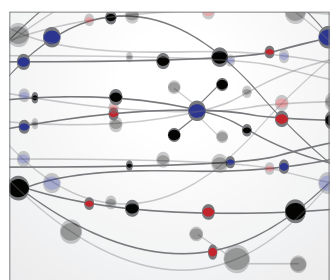

\section{The Scientific} World Journal
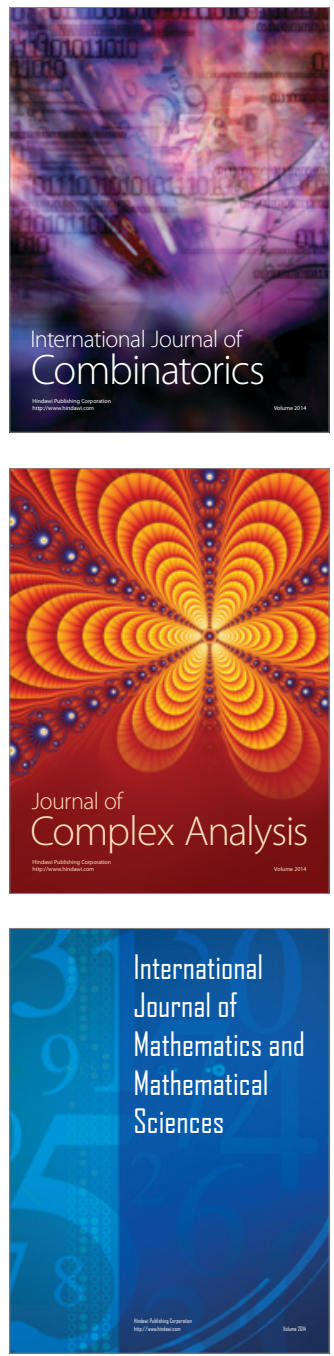
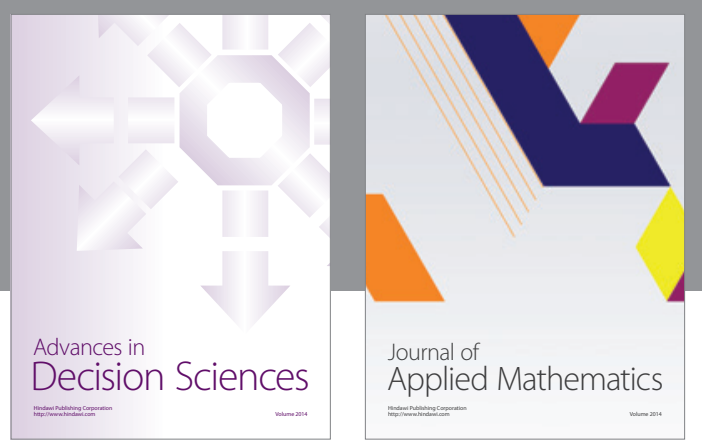

Algebra

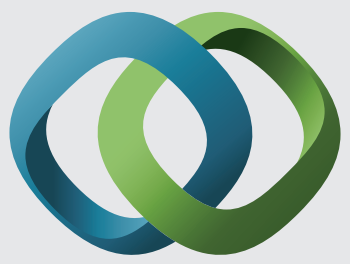

\section{Hindawi}

Submit your manuscripts at

https://www.hindawi.com
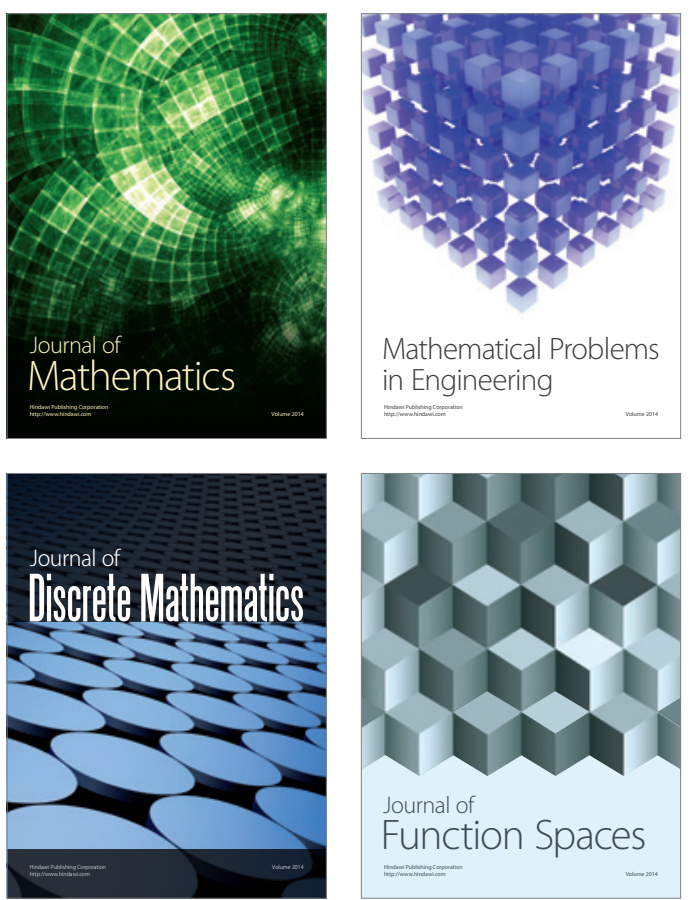

Mathematical Problems in Engineering
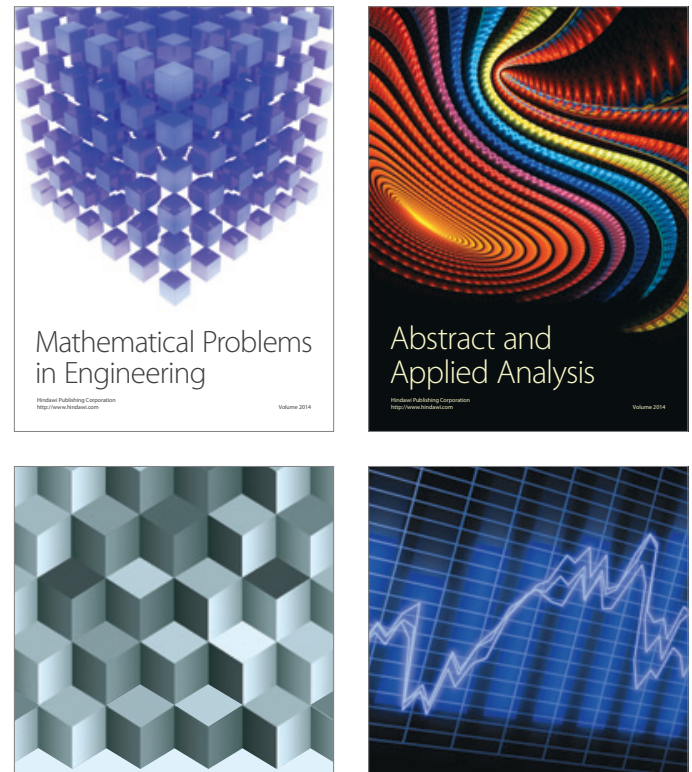

Journal of

Function Spaces

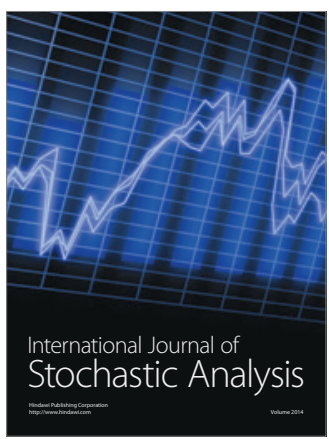

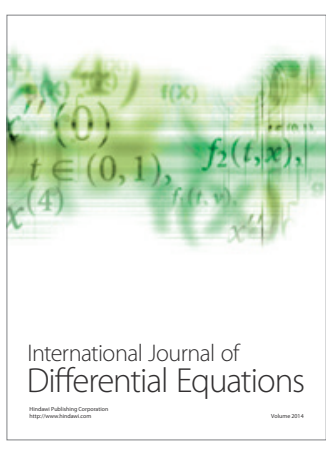
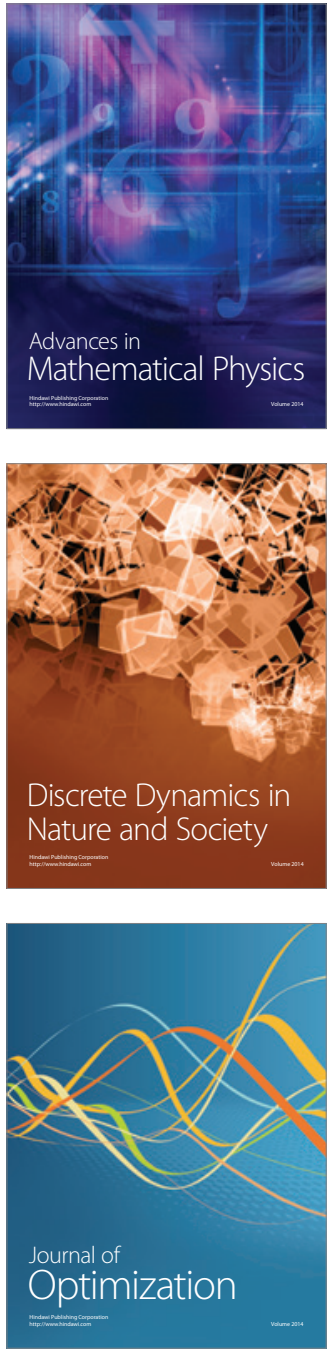